\title{
Prácticas de evaluación en Educación Infantil: un proyecto globalizado y en comunidad
}

\author{
Assessment practices in Early Childhood Education: a globalized and \\ comunity-based project
}

\author{
Beatriz Iglesias, María Colmenero \\ CPI Plurilingüe Virxe da Cela
}

\begin{abstract}
Resumen
Se presenta un estudio de caso llevado a cabo en las aulas de Educación Infantil del Centro Público Integrado (CPI) Plurilingüe Virxe da Cela en el curso académico 2016/2017, y las líneas generales de un proyecto globalizado cuyos ejes han sido las diferentes técnicas de evaluación posibles desde una secuencia didáctica que involucra dos cursos diferentes de la Educación Infantil, a la comunidad educativa y a otro agente como es la universidad. Se expone el marco curricular en el que se integra este proyecto y sus características esenciales, así como las diferentes técnicas, su justificación teórica y práctica en base a las últimas investigaciones, y ejemplos de cómo se han utilizado en este proyecto. Finalmente se detallan los resultados más llamativos en relación al proceso de enseñanza y aprendizaje para todos los involucrados. Se concluye con la repercusión que posee el tomar la evaluación y su cometido como eje en el proceso tanto de enseñanza y aprendizaje desde mucho antes incluso de poner en práctica cualquier proyecto, con la máxima de que el alumnado conozca sus capacidades, sea consciente de ellas y aprenda a ver la evaluación como una técnica de aprendizaje y proceso, no solo de resultado.

Palabras clave: instrumentos, educación infantil, evaluación, aprender a aprender, proyecto globalizado.
\end{abstract}

\footnotetext{
Abstract

English abstract. We present a case study carried out in the classrooms of Early Childhood Education in Colegio Público Integrado (CPI) Plurilingual Virxe da Cela in the academic year 2016/2017, and the general lines of a globalized project whose axes have been the different techniques of assessment from a didactic sequence that involves two different courses of Early Childhood Education, the educational community and another agent such as the university. It presents the curricular framework in which this project is integrated and its essential characteristics, as well as the different assessment techniques, their theoretical and practical justification based on the latest research, and examples of how they have been used in this project. Finally, we detail the most striking results in relation to the teaching and learning process for all those involved. It concludes with the repercussion of taking the assessment and its role as the axis in the process of teaching and learning because all students know their skills, be aware of them and learn to
}

see assessment as a learning and process technique, not just outcome.

Keywords: instruments, Early Childhood Education, assessment, learning to learn, globalized project.

\section{Introducción}

A día de hoy, es indiscutible la importancia del desarrollo de la evaluación en las aulas de Educación Infantil, así como también la necesidad de instrumentos, técnicas y modelos que ayuden a los/las docentes a su análisis, comprensión, interpretación y puesta en práctica. Aún así, estudios bibliométricos actuales destacan la todavía escasa presencia de un marco ya no tanto teórico, pero si práctico sobre la evaluación, disponiendo de un número muy limitado de instrumentos para estas primeras edades que cumplan criterios de adaptabilidad, constructivismo, atención a la diversidad y contextualizados, máximas de cualquier práctica educativa en el momento actual.

En base a ello, presentamos un estudio de caso llevado a cabo en las aulas de Educación Infantil del CPI Plurilingüe Virxe da Cela en el curso académico 2016/2017, así como las líneas generales de un proyecto globalizado cuyos ejes han sido las diferentes técnicas de evaluación posibles desde una secuencia didáctica sobre la comunidad indígena de los Masai que involucra dos cursos diferentes de la Educación Infantil, a la comunidad educativa y a otro agente como es la universidad. Así, tras un marco teórico que exponga la importancia, características y las flamantes formas y técnicas de evaluación para los diferentes momentos y en base a la competencia de aprender a aprender, se expone el marco curricular en el que se integra este proyecto y sus características esenciales. Más adelante, se expondrán las diferentes técnicas, su justificación teórica y práctica en base a las últimas investigaciones, y ejemplos de cómo se han utilizado en este proyecto, entre las que cabe destacar: la lluvia de ideas, preguntas ocultas, respuesta por pares a través del trabajo intergeneracional, la robótica como técnica de evaluación final, la pregunta metacognitiva, la asamblea, entre otras. 
Junto con la descripción de cada uno de las técnicas y formas de evaluación utilizadas, se detallan los resultados más llamativos en relación al proceso de enseñanza y aprendizaje para todos los involucrados. Se concluye con la repercusión que posee el tomar la evaluación y su cometido como eje en el proceso tanto de enseñanza y aprendizaje desde mucho antes incluso de poner en práctica cualquier proyecto, con la máxima de que el alumnado conozca sus capacidades, sea consciente de ellas y aprenda a ver la evaluación como una técnica de aprendizaje y proceso, no solo de resultado.

\section{Ejes teóricos}

El concepto de evaluación parece situarse siempre en las prácticas docentes en los momentos finales, de remate de cualquier quehacer o secuencia didáctica. Pero muy lejos de la realidad, abarca un gran número de momentos, tipologías y técnicas que favorezcan un proceso de aprendizaje en los niños y niñas en estas primeras edades que permiten una concepción y naturaleza holística y transversal de la evaluación. Las consideraciones iniciales del proyecto que se presentan se recogen en los siguientes subepígrafes.

\section{La evaluación como proceso de enseñar y aprender}

Actualmente existe un afán incansable de evaluar muchos de los aspectos del proceso de enseñanza y aprendizaje. Iglesias (2008) resalta en sus estudios la existencia de instrumentos para evaluar productos en relación al aprendizaje, pero en los últimos tiempos empieza a emerger consideraciones flamantes de la evaluación, ya no solo como un proceso de conocer resultados, si no procesos. De ahí surge el siguiente planteamiento: ¿qué técnicas de evaluación existen y se pueden utilizar en cualquier momento de la enseñanza y el aprendizaje?

Por ello, a continuación se exponen algunas de las técnicas de evaluación utilizadas a lo largo de la secuencia didáctica como instrumento de acercamiento a las capacidades del alumnado, de su proceso de aprendizaje, y como técnica de observación para el docente. Se utiliza la siguiente clasificación: instrumentos de observación, conversacional y documental (Páramo, Martínez y Raposo, 2015).

Instrumentos de observación. Distinguimos el análisis de la fotografía a través de la observación y la fotovoz (Doval, Martínez y Raposo, 2013).

Instrumentos conversacionales. Señalamos la técnica de brainstorming, como la lluvia de ideas como evaluación inicial y punto de partida de conocimientos previos; la pregunta metacognitiva del proceso de aprendizaje, qué sabemos, qué queremos saber, cómo lo vamos a hacer y qué aprendimos como las cuatro grandes columnas y ejes del proyecto globalizado; la respuesta por pares como técnica de evaluación continua distinguiendo el trabajo por parejas, en gran grupo y pequeño grupo heterogéneas en relación a la edad, capacidades y ritmos. También recogemos en esta taxonomía la asamblea como la técnica para evaluar y valorar lo que se está aprendiendo; y finalmente la robótica con la utilización de los robots Escornabot como motivación del proceso final del proyecto con un panel de respuestas.

Instrumentos documentales. Y por último citar la técnica de evaluación del análisis de contenido de las producciones, grabaciones e intervenciones de todos los agentes implicados.

Hablamos de una evaluación y un proyexto que se fundamenta en la metodología de Aprendizaje basado en en Problemas, que permite al alumnado a llegar a la solución de las preguntas que se hacen sobre el aprendizaje mediante la investigación y la reflexión, ayudando al niño y niña a desarrollar sus diferentes capacidades y competencias.

\section{El sentido de comunidad}

Uno de los fines educativos que recogen los textos legislativos y concretamente también los documentos de este centro es el sentido de la comunidad. Por ello, y en consonancia con Traver, Sales y Moliner (2010), se resalta la importancia de que un proyecto globalizado y bajo estas premisas tiene y debe contar con la participación no solo del alumnado y docentes de las aulas implicadas, sino de los demás agentes que conforman la comunidad educativa. Esto es así por los siguientes motivos: 1) dar a conocer al resto de comunidad educativa de lo que se hace en las aulas; 2) hacerlos partícipes del quehacer educativo; 3 ) conocer la visión y vivencias de la comunidad educativa relacionadas con la temática del proyecto; y 4) crear conciencia de comunidad.

Esto está estrechamente relacionado con la educación para el desarrollo, entendida como aquella educación que permite partir de las necesidades, fortalezas, intereses y características del entorno más próximo para trabajar conceptos relacionados con el mundo y la globalidad, y favorece y cuyo eje principal es el desarrollo de las capacidades críticas y reflexivas que se nos presuponen en esta sociedad.

\section{Desarrollo del proyecto}

En el siguiente epígrafe se describe el punto de partida del proyecto, dado que se enmarca en un plan de centro y aula más amplio; las características contextuales, referidas al aula y que justifican la necesidad de dicho material; el contexto curricular, que incluye los objetivos, contenidos, competencias y educación en valores que toma el proyecto, así como unas orientaciones didácticas generales; y el banco de actividades y materiales, en el que se recoge y detalla cada recurso con su tarea correspondiente.

\section{Punto de partida}

Este proyecto y su material parte de la filosofía y características del centro recogidos en el Proyecto Educativo que se base en la enseñanza y aprendizaje globalizado y con ápices constructivistas. Esto supuso que desde la Educación Infantil se diese respuesta a los siguientes principios de atención a la diversidad, globalización, individualización, ritmos diferentes, sentido de comunidad, entre otros, con prácticas educativas que intentasen responder a éstas desde una visión de la educación como algo compartido, tras un 
análisis en profundidad de las aulas y sus necesidades de dos aulas en concreto. Así, nace un proyecto globalizado conjunto entre las aulas de $5^{\circ}$ y $6^{\circ}$ de Educación Infantil del colegio CPI Plurilingüe Virxe da Cela (Monfero, A Coruña). Por ello, en términos de investigación, el estudio de caso (Hernández, Fernández y Baptista, 2010) responde a los objetivos que se plantean. Éstos pasaban por:

- Reflexionar sobre la importancia de la evaluación desde la práctica educativa en Educación Infantil.

- Crear en colaboración con el alumnado un proyecto trimestral de aula.

- Realizar e incorporar en las aulas dicho proyecto en colaboración con la comunidad educativa.

- Potenciar la capacidad de decisión propia de cada alumno/a en relación al desarrollo del proyecto según necesidades e intereses.

- Potenciar la capacidad de decisión propia de cada alumno/a en relación al desarrollo del proyecto según necesidades e intereses.

- Descubrir y dar a conocer una cultura indígena y la comparación con nuestro entorno.

\section{Características contextuales}

El centro, dependiente de la Consellería de Educación de Galicia, está situado en el Concello de Monfero, en un entorno rural que ofrece múltiples posibilidades.

Desde el centro se vienen realizando actividades en estos últimos cinco años relacionados con el contorno más próximo y bajo principios de prácticas locales. La necesidad de elaborar un proyecto de estas características derivó de un primer análisis de los documentos de centro y de aula en el que se detectó la importancia de contenidos y prácticas educativas desde una dimensión global de la educación para el desarrollo.

\section{Contexto Curricular}

El Decreto 330/2009 de 4 de junio por el que se establece el currículum de la Educación Infantil en la Comunidad Autónoma de Galicia (España) dispone tres áreas curriculares. Éste proyecto, que presenta un enfoque globalizador, se sitúa más concretamente en la de "Conocimiento del entorno". Los objetivos y contenidos de cada una de ellas quedan reflejados en la tabla 1 .
Tabla 1.

Objetivos y contenidos curriculares

\begin{tabular}{|c|c|c|}
\hline Áreas & Objetivos & Contenidos \\
\hline $\begin{array}{l}\text { Conocimiento } \\
\text { de sí mismo y } \\
\text { autonomía } \\
\text { personal }\end{array}$ & $\begin{array}{l}\text { Adquirir destrezas y } \\
\text { habilidades } \\
\text { manipulativas } \\
\text { aplicándolas al } \\
\text { manejo de útiles y } \\
\text { materiales presentes } \\
\text { en su entorno. }\end{array}$ & $\begin{array}{c}\text { Potenciación de las } \\
\text { destrezas y habilidades } \\
\text { en la manipulación de } \\
\text { instrumentos, } \\
\text { herramientas y } \\
\text { materiales. }\end{array}$ \\
\hline $\begin{array}{l}\text { Conocimiento } \\
\text { del entorno }\end{array}$ & $\begin{array}{c}\text { Establecer } \\
\text { relaciones de } \\
\text { confianza, afecto, } \\
\text { colaboración y } \\
\text { pertenencia } \\
\text { basándolas en el } \\
\text { respeto a las } \\
\text { personas y a las } \\
\text { normas de la } \\
\text { sociedad. } \\
\text { Identificar las } \\
\text { funciones de los } \\
\text { grupos y de las } \\
\text { organizaciones de la } \\
\text { comunidad } \\
\text { descubriendo las } \\
\text { principales } \\
\text { características y } \\
\text { actividades de las } \\
\text { personas que las } \\
\text { conforman. }\end{array}$ & $\begin{array}{c}\text { Conocimientos } \\
\text { diversos sobre algunas } \\
\text { profesiones y } \\
\text { servicios: } \\
\text { herramientas, } \\
\text { vestuario y espacios } \\
\text { donde desarrollan su } \\
\text { actividad, siempre con } \\
\text { una perspectiva } \\
\text { coeducativa y evitando } \\
\text { estereotipos sexistas. } \\
\text { Interés y curiosidad } \\
\text { por los } \\
\text { acontecimientos que } \\
\text { suceden a su alrededor. }\end{array}$ \\
\hline $\begin{array}{l}\text { Lenguajes: } \\
\text { Comunicación } \\
\text { y } \\
\text { representación }\end{array}$ & $\begin{array}{l}\text { Utilizar los diversos } \\
\text { lenguajes } \\
\text { entendiéndolos } \\
\text { como instrumentos } \\
\text { de comunicación } \\
\text { entre las personas, } \\
\text { de expresión de las } \\
\text { ideas y sentimientos } \\
\text { y de representación } \\
\text { de la realidad. }\end{array}$ & $\begin{array}{l}\text { Observación e interés } \\
\text { por el significado de } \\
\text { signos y símbolos } \\
\text { presentes en la vida } \\
\text { cotidiana. } \\
\text { Diferenciación entre } \\
\text { las formas escritas y } \\
\text { otras formas de } \\
\text { expresión gráfica. }\end{array}$ \\
\hline
\end{tabular}

El proyecto también responde a la competencia de aprender a aprender y a la educación en valores, fomentando el acercamiento a la competencia de inteligencia y bienestar emocional, así como la capacidad crítica y de decisión.

\section{Método}

El paradigma interpretativo ampara el estudio, conformándose como un estudio descriptivo porque se nutre del análisis y reflexión a lo largo de todas sus fases y orientado a la práctica (Latorre, Del Rincón y Arnal, 1996).

Participantes. Se presenta un estudio de observación análisis, de reflexión e intervención de un aspecto concreto en un contexto determinada, derivado de las necesidades de dicho escenario. Por ello, el estudio de caso responde a los objetivos de la investigación (Hernández, et al., 2010). Así el contexto participante son dos aulas, quinto y sexto curso de Educación Infantil de un colegio público integrado de una localidad rural, Monfero (España). Son 29 niños y niñas de entre 4 y 6 
años; las docentes de esas aulas, y diferentes miembros de la comunidad educativa ${ }^{1}$.

Instrumentos. El estudio se aventura cualitativa, manteniendo correspondencia con las técnicas e instrumentos utilizados que permiten una triangulación exhaustiva entre los datos. Estos instrumentos los podemos clasificar en:

- Instrumento de investigación: la observación participante a través de rúbricas de observación, entre las que cabe destacar Modelo de evaluación interrogativo de las estrategias (Amate, 2003), Escala de observación de estrategias cognitivas y metacognitivas (Sáiz, Carbonero y Flores, 2010), y Escala de identificación de estrategias de aprendizaje en niños pequeños (Salmerón, Ortiz y Rodríguez, 2002); Escala para la evaluación del proceso de enseñanza y aprendizaje en Educación Infantil EEPEA-EI (Rodríguez, Ortiz y Blanco, 2012), Cuestionario para la identificación de estrategias de enseñanza en el profesorado (Salmerón, et al., 2002); y el Sistema de Signos para identificar las posibilidades metacognitivas de una tarea en Educación Infantil (Páramo, Raposo y Martínez, 2013);

- Instrumentos de intervención: el proyecto didáctico que recoge todos los aspectos curriculares del mismo.

\section{Procedimiento}

El proyecto se estructuró en tres fases:

1) Preparación del estudio

1.1. Revisión y búsqueda bibliográfica. Delimitación del objeto de estudio, problemática y preguntas de investigación.

1.2. Preparación del escenario. Detección de necesidades. Definición de participantes. Análisis de entorno y documento de centro

2) Desarrollo de la investigación

2.1. Elección de instrumentos. Elaboración del proyecto

2.2. Desarrollo metacognitivo del proyecto

3) Evaluación del estudio

3.1 Análisis de la situación final.

3.2 Análisis de datos recogidos: análisis descriptivo, comparativo y de contenido e interpretación de datos, propuesta de mejora, conclusiones, limitaciones y proyección del estudio.

3.4 Difusión y publicación de los resultados.

Siguiendo las orientaciones metodológicas del anexo I del citado Decreto Curricular, este proyecto responde a la perspectiva didáctica de un proceso de aprendizaje transversal e interdisciplinar, potencialidades de esta metodología de trabajo a través de proyectos (Amor y García, 2012). El ambiente de aprendizaje también toma partido en el proyecto. Se utilizan los espacios de ambas aulas así como el pasillo donde se ha creado el rincón de los Masai. Los recursos materiales disponibles en el centro para hacer efectivo el proyecto son: materiales fungibles, cámara fotográfica, mascotas del aula, etc. Los recursos humanos son: las propias docentes, una profesora de apoyo, diferentes agentes de la comunidad educativa (profesorado de Educación Secundaria, familias, universidad...). Se cuenta también con el seguimiento de la orientadora del centro para poder atender a las diferentes fortalezas y debilidades del alumnado, atendiendo a la diversidad desde este proyecto.

En relación con los tiempos, el proyecto se ha llevado a cabo durante el segundo trimestre del curso académico 2016/2017 durante tres sesiones semanales de entre 20 y 40 minutos de duración.

En cuanto al papel de los adultos, será mediador en las diferentes decisiones y votaciones en el trascurso del proyecto, facilitador de actividades, momentos y materiales. El proyecto permite diferentes tipos de agrupamiento según la actividad o momento (gran grupo, pequeño grupo, individual en cada clase específica y entre clases) en concomitancia con otros agentes, la apertura al entorno y su continuidad en las familias.

Las actividades que se plantearon, posibilitan el abordar la atención a la diversidad, ya que a partir de la premisa de que son muy variadas las capacidades de procesamiento de información del alumnado, se permite que cada niño/a se sitúe de una manera diferente ante su propio proceso de aprendizaje.

\section{Banco de actividades}

Hubo actividades específicas para cada aula y comunes, para poder responder a los diferentes ritmos. Todas ellas partieron de unas preguntas iniciales del alumnado de $6^{\circ}$ Educación. Se recoge en la siguiente tabla 2, así como el contenido principal de cada una de ellas.

\footnotetext{
${ }^{1}$ Entre ellos alguna actividad ha contado con la participación de docentes universitarios y de la ONG Solidaridad Internacional de Galicia, ya que el centro realiza con ambos el proyecto Investigando la dimensión global de la educación para el desarrollo: un estudio piloto en una escuela gallega, que cuenta con la subvención de la Xunta de Galicia.
} 
Tabla 2.

Banco de actividades

\begin{tabular}{|c|c|c|}
\hline Actividad & Común/Específica & Descripción \\
\hline $\begin{array}{l}\text { ¿Qué } \\
\text { sabemos?: } \\
\text { Recogida de } \\
\text { ideas iniciales }\end{array}$ & $5^{\circ}$ y $6^{\circ} \mathrm{EI}$ & $\begin{array}{l}\text { Realización de una } \\
\text { tormenta de ideas para } \\
\text { conocer sus ideas sobre la } \\
\text { temática. }\end{array}$ \\
\hline $\begin{array}{l}\text { ¿Qué } \\
\text { queremos } \\
\text { saber?: } \\
\text { Preguntas } \\
\text { iniciales }\end{array}$ & $5^{\circ}$ y $6^{\circ} \mathrm{EI}$ & $\begin{array}{l}\text { Recogida de los } \\
\text { principales intereses sobre } \\
\text { los Masai del alumnado }\end{array}$ \\
\hline $\begin{array}{l}\text { ¿Dónde viven } \\
\text { los Masai? } \\
\text { ¿Cuántos son? }\end{array}$ & $6^{\circ} \mathrm{EI}$ & $\begin{array}{l}\text { Localización en un mapa } \\
\text { de África los principales } \\
\text { asentamientos Masai. } \\
\text { Búsqueda del número de } \\
\text { habitantes en internet. }\end{array}$ \\
\hline $\begin{array}{l}\text { ¿Qué comen y } \\
\text { que beben los } \\
\text { Masai? }\end{array}$ & $5^{\circ}$ y $6^{\circ} \mathrm{EI}$ & $\begin{array}{l}\text { Uso del lenguaje escrito } \\
\text { para la respuesta de ambas } \\
\text { preguntas. }\end{array}$ \\
\hline $\begin{array}{l}\text { Abalorios } \\
\text { Masai }\end{array}$ & $5^{\circ}$ y $6^{\circ} \mathrm{EI}$ & $\begin{array}{l}\text { Elaboración de collares } \\
\text { con materiales reciclados. } \\
\text { Uso de técnicas artísticas } \\
\text { sobre papel. }\end{array}$ \\
\hline $\begin{array}{l}\text { Vestimenta } \\
\text { Masai }\end{array}$ & $5^{\circ}$ y $6^{\circ} \mathrm{EI}$ & $\begin{array}{l}\text { Creación de vestimenta } \\
\text { Masai. Expresión plástica } \\
\text { de sus vestimentas. }\end{array}$ \\
\hline $\begin{array}{lr}\text { Visionado } & y \\
\text { análisis } & \text { de } \\
\text { fotografías } & \end{array}$ & $5^{\circ}$ y $6^{\circ} \mathrm{EI}$ & $\begin{array}{l}\text { Charla de una profesora de } \\
\text { Ed. Secundaria sobre su } \\
\text { viaje a Kenia. Análisis de } \\
\text { sus fotografías. }\end{array}$ \\
\hline $\begin{array}{l}\text { Hogares } \\
\text { Masai }\end{array}$ & $5^{\circ}$ y $6^{\circ} \mathrm{EI}$ & $\begin{array}{l}\text { Creación mediante barro y } \\
\text { elementos naturales de las } \\
\text { chabolas Masai. }\end{array}$ \\
\hline La tribu & $5^{\circ}$ y $6^{\circ} \mathrm{EI}$ & $\begin{array}{l}\text { Uso del lenguaje escrito y } \\
\text { plástico para establecer las } \\
\text { distintas funciones que } \\
\text { realizan los miembros de } \\
\text { las tribus Masai. }\end{array}$ \\
\hline Fauna & $6^{\circ} \mathrm{EI}$ & $\begin{array}{l}\text { Empleo de diferentes } \\
\text { técnicas plásticas para el } \\
\text { conocimiento de la fauna } \\
\text { africana. }\end{array}$ \\
\hline Flora & $5^{\circ} \mathrm{EI}$ & $\begin{array}{l}\text { Conocimiento de los } \\
\text { distintos tipos de paisajes } \\
\text { empleando de la mesa de } \\
\text { luz. }\end{array}$ \\
\hline Lengua Maa & $6^{\circ} \mathrm{EI}$ & $\begin{array}{ll}\text { Aprendizaje } & \mathrm{de} \\
\text { vocabulario Maa } & \end{array}$ \\
\hline Escudos & $5^{\circ}$ y $6^{\circ} \mathrm{EI}$ & $\begin{array}{l}\text { Elaboración de escudos } \\
\text { Masai. }\end{array}$ \\
\hline $\begin{array}{ll}\text { ¿Por } & \text { qué } \\
\text { pastorean } & \text { las } \\
\text { vacas? } & \end{array}$ & $5^{\circ} \mathrm{EI}$ & $\begin{array}{l}\text { Uso de acuarela y pincel } \\
\text { para el aprendizaje de las } \\
\text { funciones de los pastores. }\end{array}$ \\
\hline $\begin{array}{l}\text { ¿Cómo } \\
\text { hacen } \\
\text { mayores? }\end{array}$ & $5^{\circ} \mathrm{EI}$ & $\begin{array}{l}\text { Impregnación en papel de } \\
\text { materiales plásticos para } \\
\text { dar respuesta a la } \\
\text { pregunta. }\end{array}$ \\
\hline El carnaval & $5^{\circ}$ y $6^{\circ} \mathrm{EI}$ & Baile y caracterización. \\
\hline
\end{tabular}

\section{Primeros resultados y conclusiones}

Los primeros resultados tras el análisis de contenido de las diferentes actividades, así como de las escalas a utilizar nos permiten realizar las siguientes consideraciones iniciales: 1) el mayor interés del alumnado en las actividades que parten de la evaluación como eje transversal; 2) datos que revierten un proceso de aprendizaje más metacognitivo por parte de todos los implicados; 3) y la importancia de crear rúbricas que permitan la recogida de datos en cada actividad.

El proyecto realizado posibilitó:

- Al profesorado:

o Reflexionar sobre la práctica educativa y la importancia de la evaluación

o Potenciar la metacognición a través del ambiente, momentos y tiempos.

- Al alumnado:

o Conocer y usar sus capacidades para aprender.

o Valorar sus sentidos para el desarrollo de las capacidades que posibilitan el aprender a aprender.

- A la comunidad educativa:

o Participar y conocer un proyecto y quehacer educativo

Todo este entramado de elementos de investigación le confiere al estudio un carácter descriptivo y reflexivo con el que pretendemos, conscientes de sus salvedades, llevar a cabo un proyecto interdisciplinar en un aula de Educación Infantil, como primer eslabón para desplegar todas las posibilidades de la evaluación como técnica de aprendizaje que respete los ritmos y capacidades de todos los implicados.

La prospectiva del estudio se situará en analizar exhaustivamente los datos y analizar el proceso de enseñanza y aprendizaje y sus posibilidades metacognitivas, así como la elaboración de cuestionarios para los diferentes participantes, para conocer su opinión sobre el mismo. Y por otro lado, la creación y puesta en marcha de otro tipo de actividades donde la participación de la comunidad educativa sea mayor.

En definitiva, se realizó un proyecto que permitiese a todos los implicados unas primeras pinceladas sobre las múltiples posibilidades de la evaluación y sus diferentes técnicas para situarnos en una práctica educativa con indicios de proyecto basado en problemas, trabajo cooperativo y desde la participación de la comunidad educativa.

\section{Agradecimientos}

Al CPI Plurilingüe Virxe da Cela por dejarnos crecer como maestras.

\section{Referencias}

Amate, J. (2003). Procedimiento para la evaluación de las estrategias de autorregulación durante el aprendizaje en Educación Infantil. Revista Electrónica de Investigación Psicoeducativa y Psicopedagógica, 1 (1), 19-42.

Amor, A. y García, M.T. (2012). Trabajar por proyectos en el aua. Revista Latinoamericana de Educación Infantil,

1

(1),

127-155. 
http://redaberta.usc.es/reladei/documentos/volumenes/ volumen1_n1_completo.pdf.

Doval, M.I., Martinez-Figueira, M.E. y Raposo-Rivas, M. (2013). La voz de sus ojos: la participación de los escolares mediante Fotovoz. Revista de investigación en educación, $11 \quad$ (3), 150-171. http://reined.webs.uvigo.es/ojs/index.php/reined/articl e/viewFile/741/308

Galicia. Decreto 330/2009 de 4 de Junio, junio por el que se establece el currículum de la Educación Infantil en la Comunidad Autónoma de Galicia. DOG no 121 , de 23 de Junio de 2009.

Hernández, R., Fernández, C. y Baptista, P. (2010). Metodología de la investigación. México: McGraw-Hill.

Iglesias, M. L. (2008). Observación y evaluación del ambiente de aprendizaje en Educación Infantil: dimensiones y variables. Revista Iberoamericana de Educación, 47, 49-70.

http://www. rieoei.org/rie47a03.pdf

Latorre, A., Del Rincón, D. y Arnal, J. (1996). Bases metodológicas de la Investigación Educativa. Barcelona: GR 92.

Páramo, M. B., Martínez-Figueira, M.E. \& Raposo-Rivas, M. (2015). Instrumentos de una investigación en desarrollo sobre el aprender a aprender: descripción y posibilidades. Revista de Estudios e Investigación en Psicología y Educación, 6 (extraordinario), 66-71.

Páramo, M.B., Raposo, M. y Martínez, M.E. (2013). Una educación infantil inclusiva y metacognitiva puesta en interrogantes: diseño de una investigación basada en organizadores hipertextuales. En B. D. Silva, L.S. Almeida, A. Barca, M. Peralbo, A. Franco y R. Monghino, Atas do Congresso Internacional Galego Portugués de Psicopedagogía. Braga: CIE-d. 6852-6863.

Rodríguez, S., Ortiz, L., \& Blanco, M.E. (2012). Percepciones del profesorado de Educación Infantil sobre sus propuestas de enseñanza en aprendizaje autorregulado. Profesorado, 16 (1), 143-164.

Sáiz, M.C., Carbonero, M.Á., y Flores, V. (2010). Análisis del procesamiento en tareas tradicionalmente cognitivas y de teoría de la mente en niños de 4 y 5 años. Psicothema, 22 (4), 772-777.

Salmerón, H., Ortiz, L., y Rodríguez, S. (2002). Identificación de estrategias de aprendizaje en educación infantil y primaria: propuesta de instrumentos. Revista Española de Orientación y Psicopedagogía, 13 (1), 89-106.

Traver, J.A., Sales, A., y Moliner, O. (2010). Ampliando el territorio: algunas claves sobre la participación de la comunidad educativa. Revista Iberoamericana sobre Calidad, Eficacia y Cambio en Educación, 8 (3), 97-119 http://www.redalyc.org/html/551/55115052007/. 